Itinéraires Itinéraires

Littérature, textes, cultures

2016-2 | 2017

Livre, sérialité et transmédialité

\title{
Du Chaka de Thomas Mofolo au Zulu de Tchicaya U Tam'si, le feuilleton radiophonique comme étape de la réécriture
}

Between Thomas Mofolo's Chaka and Tchicaya U Tam'si's Zulu, the Radio Series as a Preparation for Drama

Pierre Leroux

\section{OpenEdition}

Journals

Édition électronique

URL : http://journals.openedition.org/itineraires/3404

DOI : 10.4000/itineraires.3404

ISSN : 2427-920X

Éditeur

Pléiade

Référence électronique

Pierre Leroux « Du Chaka de Thomas Mofolo au Zulu de Tchicaya U Tam'si, le feuilleton radiophonique comme étape de la réécriture », Itinéraires [En ligne], 2016-2 | 2017, mis en ligne le 25 avril 2017, consulté le 01 octobre 2020. URL : http://journals.openedition.org/itineraires/3404 ; DOI : https:// doi.org/10.4000/itineraires.3404

Ce document a été généré automatiquement le 1 octobre 2020.

\section{(c) $($ ) $\odot \odot$}

Itinéraires est mis à disposition selon les termes de la licence Creative Commons Attribution - Pas d'Utilisation Commerciale - Pas de Modification 4.0 International. 


\title{
Du Chaka de Thomas Mofolo au Zulu de Tchicaya U Tam'si, le feuilleton radiophonique comme étape de la réécriture
}

\author{
Between Thomas Mofolo's Chaka and Tchicaya U Tam'si's Zulu, the Radio Series \\ as a Preparation for Drama
}

Pierre Leroux

1 Gérald Félix-Tchicaya, plus connu sous le nom de plume de Tchicaya U Tam'si, est un poète, romancier et dramaturge congolais (1931-1988). Fils du premier député du Moyen-Congo, Jean-Félix Tchicaya, il arrive en France en 1946 et y passera la plus grande partie de sa vie. Il publie son premier recueil, Le Mauvais Sang en 1955 et entre en 1957 à la radiodiffusion de la France d'Outre-mer, ancêtre de RFI. Il y restera jusqu'en 1960 et participera notamment à l'émission Ici Paris, Courrier Africain. Après un bref passage par Léopoldville au moment de l'indépendance du Congo belge, il suit une carrière de diplomate à l'UNESCO.

2 La création radiophonique est marquée par la dramatisation d'un certain nombre de contes avec laquelle la production de l'anthologie Légendes africaines en $1967 \mathrm{chez}$ Seghers ne sera pas sans lien. Ce travail, tout comme des entretiens et des chroniques littéraires, s'inscrit déjà dans la volonté de faire œuvre et de s'approprier des matériaux divers: "J'ai pris treize légendes et même des fragments de légendes, pour en faire une légende, un peu comme la très fameuse légende des siècles ${ }^{1}$.» En 1958, c'est un classique de la littérature sud-africaine, Je ne suis pas un homme libre de Peter Abrahams, qui est adapté et présenté aux auditeurs par Tchicaya. Parmi toutes ces productions qui puisent dans les littératures écrites et orales, le feuilleton

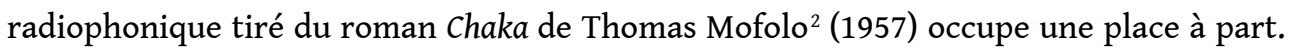
En effet, il s'agit là d'une série dans laquelle on retrouve une partie de l'équipe qui 
anime Ici Paris, Courrier Africain. Les animateurs Douta Seck et Pierre de Georgi jouent ainsi deux des principaux rôles et Michel Moitessier assure la réalisation.

Ce principe qui consiste à reprendre de grands titres de la littérature pour en faire des feuilletons est loin d'être exceptionnel à cette époque: aussi peut-on relever une adaptation de Vidocq en vingt épisodes (Gével 1959) ou une relecture de la Comédie humaine (Lucien de Rubempré, tricheur romantique) en cent quinze épisodes (Brest et Weitzmann 1963). Ce type d'exercice joue sur la notion de sérialité, notamment si l'on considère le mode de diffusion en feuilleton des romans de Balzac. Il y aurait donc pour ces œuvres une dialectique entre la publication en livraisons, la lecture en volume et une nouvelle fragmentation de l'intrigue pour la radio.

列 éducative et volonté de plaire, mais il ajoute encore un niveau de complexité car il entre en résonance avec d'autres représentations du roi zoulou. En effet, il n'est pas le seul à adapter ce roman. De Senghor en $1951^{3}$ au Togolais Zinsou en 1972, et même audelà, on compte une bonne dizaine d'adaptations théâtrales, rien que dans le domaine francophone. De plus, Tchicaya adaptera lui-même son feuilleton pour en faire la pièce Le Zulu créée à Avignon en 1976.

5 La dimension radiophonique de l'œuvre de Tchicaya U Tam'si a suscité peu d'analyses, tant du côté de la critique que de l'auteur lui-même. Elle est cependant loin d'être anecdotique, comme le dénote l'une des lettres qu'il adresse à Michel Vincent. Cette dernière contient un commentaire qui porte sur le travail de producteur radio en général et son influence sur la production ultérieure :

Si le travail radiophonique a influencé ma production écrite? Cela est certain. Pas d'une manière que je jugerais positive. La différence se lit entre la manière des Cancrelats et celle des Méduses. Ici de longues séquences - là des plans courts. Ici encore le foisonnement, l'éparpillement. Là encore une certaine rapidité du trait ${ }^{4}$. (Cité dans Vincent 1994 : 13)

Il est d'ailleurs important ici de préciser que Les Cancrelats, publié en 1980 a été rédigé dès les années 1950 selon des sources concordantes. Il y aurait donc une manière d'écrire le roman avant la radio et une autre après. Dans le cas de Chaka, le phénomène est plus saillant car c'est la même histoire qui est traitée, avec des changements significatifs, à vingt ans d'écart.

7 Cette complexe configuration intertextuelle invite à examiner l'amont et l'aval de la pièce radiophonique pour mieux rendre compte de son contexte de production et de l'importance qu'elle a eue pour la suite de la carrière de Tchicaya. En d'autres termes, nous nous demanderons quelle place occupe le travail du dramaturge congolais dans le réseau intertextuel tissé autour de la figure de Chaka avant de nous arrêter sur le rôle joué par le feuilleton dans la genèse du Zulu.

\section{La postérité du roi zoulou}

8 Chaka, il faut le préciser, est avant tout un personnage historique (1788-1828) et le fondateur de l'Empire zoulou, dans ce qui est aujourd'hui la partie est de l'Afrique du Sud (région de Durban). S'il faut chercher un point d'origine à sa postérité littéraire, celui-ci se trouve certainement dans les Izibongo, ces chants de louange composés de son vivant et compilés au xxe siècle par Trevor Cope (1968). À partir de là, ce qui frappe dans la constitution de cette figure littéraire, c'est la variété des sources et la 
multiplication des interprétations proposées. La répartition entre une légende noire et une légende dorée proposée par Huenumadji Afan $(2006)^{5}$ est intéressante mais elle est loin de résumer toutes les contradictions de ces différents Chaka. Le critique anglophone Donald Burness, pour sa part, nous offre une cartographie des représentations qui situe les différents visages du roi sur le continent africain :

Le mythe de Chaka dans la littérature africaine n'est pas homogène. Ce constat va de soi si l'on considère que le roi zoulou est représenté avant tout comme une figure romantique en Afrique francophone, comme une figure militaire en Afrique du Sud, et est totalement inconnu en Afrique de l'Est et parmi les pamphlétaires du Onitsha Market au Nigeria ${ }^{6}$. (Burness $1976: 13$ )

9 Cette répartition s'explique sans doute en partie par l'éloignement progressif des lieux du drame. Le Lifaqané, période de troubles résultant des conquêtes de Chaka, a entraîné d'importants déplacements de population au début du $\mathrm{xIX}^{\mathrm{e}}$ siècle. L'implantation ndebele dans l'actuel Zimbabwe résulte ainsi de la défection d'un général zoulou, Oumsélé Katsi. Bien souvent, dans la littérature de la région, Chaka n'est plus un héros mais un monstre assoiffé de sang.

Pour bien comprendre le contexte de production du feuilleton radiophonique et de la pièce de Tchicaya, il est nécessaire de se pencher plus en détail sur la figure « romantique » qui domine l'Afrique francophone. Le double article de Mongo Beti sur Chaka en littérature et dans l'histoire témoigne ainsi de l'importance de ce personnage pour les écrivains africains de langue française. Sa conclusion résume toute l'ambiguïté de ce roi qui régna sans partage pendant douze ans :

Qui fut au juste Chaka? Un Alexandre assoiffé de prouesses et de conquêtes? Un dictateur sanguinaire, sorte d'archétype du tyran noir, qu'allaient illustrer cent cinquante ans plus tard les Duvalier, Idi Amin Dada, Sékou Touré, Ahmadou Ahidjo, Bokassa, Mobutu? L'historiographie noire le démêlera peut-être avec le temps. (Beti et Tobner 1989: 64)

Pour le Malien Djibril Tamsir Niane (1971) ou le Sénégalais Abdou Anta Kâ (1972), l'éloignement géographique permet de faire de Chaka un héros panafricain qui, contrairement au Mandingue Soundjata ou au Toucouleur El Hadj Omar, peut servir de modèle à tout Malien ou tout Sénégalais, quelle que soit son origine ethnique.

L'autre différence est à chercher du côté de la documentation utilisée. Si les sources revendiquées par les auteurs anglophones sont variées, les dramaturges francophones font preuve d'une remarquable cohérence-il s'agit essentiellement de pièces de théâtre. En effet, c'est le roman Chaka du Southo Thomas Mofolo qui sert d'inspiration directe ou indirecte dans la plupart des cas. Ce texte publié en 1925 est d'abord traduit en anglais en 1931 avant de connaître une version française dès $1940^{7}$. Cette traduction, et plus tard le poème dramatique de Senghor, contribueront à forger de nouvelles images de Chaka dès les années 1950.

13 Dans l'épopée bantoue de Mofolo, le souverain est un être ambigu, brimé dans sa jeunesse et marqué par le sceau d'un péché originel : la relation hors mariage de son père Senza'ngakonna et de sa mère Nandi. Cette culpabilité dont hérite le jeune homme est présentée dans le roman à plusieurs reprises :

Combien amer est le fruit du péché! Voilà, à cause de son péché, Senza'ngakona obligé de se séparer de la femme qu'il aime, celle qu'il a épousée en donnant pour elle cinquante-cinq têtes de bétail, obligé aussi d'être séparé de Chaka, le premier de ses fils, et pour comble forcé de leur rendre la vie difficile. (Mofolo [1940] 1990 : 37) 
14 d'un prisme chrétien. Il est question d'une époque lointaine, d'un temps d'avant les missions « où les hommes vivaient dans les ténèbres" (Mofolo [1940] 1990 : 307). Il invente les personnages du sorcier Issanoussi et de ses aides Ndlébe et Malounga qui mèneront le jeune roi à sa perte et représentent l'influence satanique du paganisme.

Senghor, quant à lui, justifie les horreurs commises par Chaka comme un mal nécessaire et transforme le tyran en héros incompris et en Christ «cloué au sol par trois sagaies » ([1951] $2007: 118)$ entre « deux larrons ». La violence devient pour lui un moyen et non une fin en soi et même le meurtre de sa fiancée, Noliwé, n'est plus un crime mais un renoncement : «Je ne l'aurais pas tuée si moins aimée » (Ibid. : 121).

Pour mieux comprendre l'impact de Mofolo sur la tradition francophone, prenons deux œuvres sud-africaines qui revendiquent plus ou moins directement une autre source : le journal autobiographique de Henry Francis Fynn (2004), aventurier britannique qui a passé beaucoup de temps auprès de Chaka. La série télévisée Shaka Zulu (Faure 1986) et Assegaï de Nickie McMenemy (1973) se concentrent sur la rencontre de deux mondes (homme blanc ou jeune femme métisse) alors que l'arrivée des Occidentaux n'a pas eu lieu dans le roman de Mofolo. Il ne s'agit encore que d'une prédiction faite par le roi lui-même sur son lit de mort. Cette différence de perspective est essentielle car elle explique en grande partie l'attrait exercé par le monde précolonial présenté dans le roman de Mofolo. Les auteurs francophones en quête de héros noirs ont besoin d'un Chaka pur de tout contact avec l'Occident pour montrer que l'Afrique n'était pas dépourvue de grandeur avant l'arrivée des Blancs.

\section{Fantômes et figures : variations autour de la notion de personnage}

17 Le feuilleton radiophonique de Tchicaya est explicitement une adaptation dramatisée du roman de Thomas Mofolo, ainsi que l'indique le générique pour chaque épisode. Des passages entiers sont repris tels quels et il pourrait sembler au premier abord que l'auteur congolais se contente de redécouper l'œuvre du catéchiste southo. Cela dit, en plus des remaniements imposés par le changement de support, l'auditeur familier de l'œuvre de Mofolo comprend progressivement que Tchicaya insère ici ou là des éléments personnels dont certains se retrouvent, vingt ans plus tard, dans Le Zulu.

Les hallucinations de Chaka, symptômes de sa culpabilité, occupent une place importante dans le roman de Mofolo. Dans le dernier chapitre, «La fin de Chaka », ces visions prennent le pas sur le reste et le héros aperçoit un vallon qui est «rempli des créatures humaines auquel lui, Chaka avait fait donner la mort». Sa mère et sa femme occupent une place de choix dans ce tableau horrifique : "Nandi se tenait à l'une des extrémités du vallon, Noliwè à l'autre» (Mofolo [1940] 2010 : 330). La force de ces visions repose sur le retour de personnages dont le meurtre a été particulièrement affreux et sur la démultiplication des spectres. La femme de Chaka, Noliwé, est tuée alors qu'elle est enceinte et la mère du roi, Nandi, périt pour avoir sauvé de la mort un de ses petits-fils. Ces deux femmes que Chaka a aimées avant de les faire disparaitre sont les principales tortionnaires dans les derniers chapitres. Or, il est difficile de rendre à la radio l'effet produit par un gouffre immense rempli de cadavres. Tchicaya se sert des hallucinations de son héros pour proposer une variante par rapport au texte

Itinéraires, 2016-2 | 2017 
qu'il adapte. En effet, Longani est une jeune femme, une compagne de Nandi et Noliwé, qui n'existe pas dans le roman mais joue un rôle essentiel dans le feuilleton radiophonique. Par bien des aspects, son fonctionnement est celui d'un personnagefigure $^{8}$ plus que d'un personnage-personne et elle porte sur ses épaules toute la culpabilité de son roi.

Lors de sa première apparition (épisode 15) elle pressent la mort de Noliwé qui rêve «d'attendre » sans savoir ce qu'elle «attend». Devant les autres femmes incrédules, elle fait un constat qui recoupe ce que l'auditeur sait déjà : «Chaka a tué son frère pour prendre le pouvoir. Chaka n'a encore tué personne pour prendre le pouvoir de Ding'Iswayo. » Au moment où la jeune fille s'exprime, Chaka a déjà pris la décision de sacrifier l'être qui lui est le plus cher pour atteindre le pouvoir suprême. Figure de Cassandre, Longani n'est pas crue et les dernières répliques la concernant insistent sur sa marginalité : «Elle est donc si simple qu'on le dit?» Personnage sans véritable intériorité et avant tout support de prophétie, elle reviendra en spectre et en magicienne dans la suite de la diégèse.

Dans le roman, Noliwé ignore jusqu'à l'instant fatal que son mari et amant souhaite sa mort. Dans la pièce de théâtre, la jeune femme pressent que Chaka est responsable de la mort de son frère et elle devient folle avant d'être assassinée. Le rôle de Longani dans le feuilleton radiophonique permet de combiner les deux approches car cette dernière s'adresse à Ndlébe, l'un des serviteurs qui manipulent Chaka, en se faisant passer pour Noliwé. Elle porte son accusation dans un style imagé qui n'est pas sans rappeler l'œuvre poétique de l'auteur congolais : «Je veux voir mon beau et cruel fiancé. Tu ne l'as pas vu, dis, tu n'as pas vu mon perfide bien-aimé ? Tel est mon bien-aimé, un éclair dans l'orage. Et moi je suis l'oiseau dans l'arbre qu'il foudroie. » Aussitôt démasquée par Ndlébe, elle continue pourtant son jeu de rôles en désignant le roi comme «celui que Ndlébe trompe, celui que Malounga trompe, Chaka qu'Issanoussi trompe!». Longani disparaît après avoir fait comprendre au personnage qu'elle sait tout du plan ourdi par ces proches conseillers de Chaka. Elle s'affirme à partir de ce moment comme celle qui prend en charge la voix et l'apparence des autres, une figure aussi insaisissable que la vérité du récit et le destin des personnages.

21 À l'épisode dix-huit, le personnage est de retour auprès de Nandi, la mère de Chaka, après deux ans d'absence. Elle explique qu'elle a suivi des magiciens et qu'elle $\mathrm{a}$ « appris à lire le cœur des gens ». Elle met en garde la mère de Chaka mais, contrairement à ce que nous avons vu pour l'extrait précédent, elle semble le faire sans endosser un rôle. Elle devient, par son omniscience, une sorte d'équivalent féminin du sorcier Issanoussi. La suite de l'épisode ramène pourtant Longani à son instabilité et à son inconsistance : Nandi se réveille et toute la scène qui annonce sa mort se résume pour elle à une vision ou à un mauvais rêve.

La jeune fille "simple » de l'épisode quinze perd sa réalité physique en même temps qu'elle se rapproche d'une vérité cruelle et douloureuse. Folle jouant le rôle de Noliwé ou prêtresse qui visite Nandi en songe, elle achève sa transformation à l'épisode dixneuf en accusant directement Chaka d'avoir commis plus de crimes que quiconque. Ndlébe est présent lors de cette scène mais le roi est le seul à entendre la jeune femme et il passe pour un fou écrasé par sa culpabilité :

Qui viendra à ton secours? Tu as tué ta mère, tu as tué ta femme, tu as tué ton propre sang. Les vivants ne vont pas au secours des morts, il n'y a que cette musique qui puisse te venir en aide, appelle encore, inutile, on t'a entendu. Regarde qui vient. Approche, Noliwé, Chaka est en danger, en danger de mort ! 
trairement à ce qui se passe dans le roman, Longani sert d'intermédiaire entre Chaka et les morts, elle les appelle et décrit leur arrivée. Le jeu sur la présence et l'absence du personnage fonctionne extrêmement bien lorsque la voix est seule et que l'auditeur reconstitue la scène par l'imagination.

Au théâtre, dans Le Zulu, les moyens utilisés ne peuvent être les mêmes. Longani disparaît, tout comme le devin et féticheur Issanoussi. Pour manipuler Chaka et le mener à sa perte, il ne reste plus, en dernière instance que les deux serviteurs - Ndlébe et Malounga - ainsi qu'une " Voix », personnage désincarné qui constitue peut-être un reliquat radiophonique ${ }^{9}$. Selon Kahiudi Claver Mabana (2002: 126), il s'agit là d'un élément essentiel dans l'œuvre de Tchicaya: "L'absence d'un Issanoussi désoriente complètement l'intrigue, tandis que la Voix suggère une instance de conscience et un artifice d'érudition, de sagesse et un rappel constant du destin et des oracles. »

Le feuilleton radiophonique apparaît après coup comme un laboratoire et un lieu d'apprentissage où s'élabore déjà la construction théâtrale de la figure. Contrairement au héros de Senghor, le Chaka de Tchicaya, lorsqu'il monte sur les planches, échappe en grande partie à la dimension christique. Il est plutôt l'homme du mauvais sang, le barbare rimbaldien et le voleur de feu. Il n'est pas sacrifié, trahi par ses frères comme chez Mofolo. Il se suicide et, jusqu'au bout, prend en main son propre destin sans faire passer les intérêts de son peuple avant les siens ${ }^{10}$. D'autres personnages comme Noliwé prennent cependant en charge une dimension sacrificielle et l'utilisation de formules récurrentes ou de prophéties construit un nouveau rapport aux personnages et à leurs parcours.

\section{Formules et présages, la loi des séries}

Pour Kahiudi Claver Mabana, dans son ouvrage sur le mythe de Chaka, l'utilisation de formules récurrentes est l'un des traits caractéristiques de la pièce de Tchicaya. Cet aspect est présent, quoique de manière moins insistante, dans le roman de Mofolo et le feuilleton radiophonique qui nous occupe. Le rythme créé par cette récurrence a à voir avec les changements opérés d'un genre à l'autre. Le procédé qui a une fonction essentiellement mémorielle dans les deux premiers cas prend avec la pièce de théâtre une dimension symbolique. Les personnages sont travaillés, comme nous le verrons, par ces formules qui se figent et les poussent à la folie.

Pour bien comprendre la spécificité de chacun des trois genres représentés ici, il est utile de s'arrêter sur les moyens mis en œuvre pour l'exposition de la diégèse. Dans le roman de Thomas Mofolo, un narrateur raconte l'histoire et la commente. Dans la pièce de théâtre, tout passe par des dialogues ou des monologues des personnages. Le feuilleton radiophonique - tout comme quelques autres adaptations théatrales de Chaka $^{11}$ - occupe une position médiane en choisissant de s'octroyer les services d'un conteur. Contrairement au narrateur homodiégétique de Mofolo, le conteur observe la rencontre de Chaka et d'Issanoussi lors de l'épisode six et discute même avec le jeune homme au moment où il devient chef dans l'armée de Ding'Iswayo. Alors que notre personnage a le statut de " conteur " (c'est ainsi que Chaka l'appelle) et non de devin ou de magicien comme Issanoussi et Longani, le futur roi des Zoulous ne s'étonne absolument pas que des épisodes connus de lui seul - telle la rencontre avec le Seigneur des Eaux Profondes - soient évoqués par son compagnon. Le savoir du conteur n'a

Itinéraires, 2016-2 | 2017 
aucune incidence sur le déroulement de l'intrigue. Le dispositif radiophonique rejoue sur un mode biaisé la situation d'énonciation de la littérature orale mais l'auditeur est alors coupé de l'émetteur et le montage en studio remplace la performance ${ }^{12}$.

Parmi les points communs entre le récit traditionnel et le feuilleton radiophonique, le rapport à la mémoire apparaît comme une donnée capitale. C'est dans ce cadre que répétitions et résumés prennent tout leur sens. Prenons comme exemple l'ouverture de l'épisode onze :

Résumé des épisodes précédents. Chaka, le fils de Senza N'gakonna, chef d'une tribu zouloue, a été abandonné dès l'enfance par son père. Après maintes aventures, le voici réfugié chez le grand chef Ding'Iswayo. Il se distingue dans toutes les campagnes guerrières contre Zwidé, l'important voisin de Ding'Iswayo. Le temps a passé, le temps passe et l'on vient annoncer à Ding'Iswayo la mort de Senza N'gakonna, le père de Chaka. Chaka va-t-il succéder à son père? Non. C'est son frère, M'Fokazana qui a pris la succession. Un moment, Chaka croit son avenir compromis. Avec l'appui de Ding'Iswayo, il entreprend une campagne contre son frère et revient victorieux. Sauvagement, il tue M'Fokazana et c'est à la demande de Ndebelé et Malounga ses compagnons et disciples d'Issanoussi le magicien qu'il épargne ses deux autres frères, Dingana et Malangana.

L'apposition joue bien ici son rôle de prédication seconde en position détachée, fournissant de manière synthétique des informations utiles sur les personnages principaux. Chaka est «le fils de Senza Ngakonna ", Zwidé «l'important voisin de Ding'Iswayo » et chacun se réduit à un trait de crayon. Ce passage obligé de chaque épisode qui situe l'intrigue pour l'auditeur n'est pas propre à Tchicaya. Il institue dans tout feuilleton radiophonique un va-et-vient entre l'épisode singulier et le Tout qu'il faut garder à l'esprit. Le retour de formules frappantes, parfois accompagnées d'un effet sonore, participe de la même fonction mémorielle. À la fin des épisodes trois et quatre, la même phrase, est répétée en guise de conclusion : «Salut, un fils t'est né, un veau destiné aux vautours ${ }^{13}$. » Le traducteur responsable de la version française, Victor Ellenberger, précise que cette expression imagée fait référence au destin de l'enfant: « un guerrier qui périra dans un combat et deviendra la proie des vautours ${ }^{14}$ ».

Si les formules récurrentes rythment les épisodes, les apartés sont rares dans Le Zulu et les conditions de représentation n'ont pas grand-chose à voir avec celles du feuilleton. Il est inutile de résumer sans arrêt l'intrigue et les formules qui pourtant sont omniprésentes et n'ont pas le même rôle à jouer. Trois syntagmes en particulier, constats ou prophéties, reviennent de manière obsessionnelle et semblent structurer le texte. À la scène trois, la Voix annonce de manière cryptée l'arrivée des Occidentaux : "Que rien de blanc n'apparaisse au sud... Surveille l'écume de la mer " (U Tam'si 1977 : 28). À l'acte trois, scène quatre, c'est Zwidé, trahi par Chaka, qui maudit ce dernier : " Tu trahis et tu condamnes. Moi, je te maudis. Ton propre sang t'étouffera ( (Ibid.). Enfin, Noliwé qui se doute que son mari est responsable de la mort de son frère répète jusqu'à la folie une phrase de Chaka : «Souvent le destin improvise. Je vais faire comme lui » (Ibid. : 76). Dans les deux premiers cas, les paroles alimentent les obsessions du roi, dans le dernier c'est sa femme, variation autour de l'Ophélie de Shakespeare, qui s'enferme dans les mots.

31 Noliwé souligne la démesure de Chaka qui se prend pour le destin lui-même et donc pour une force aveugle et impitoyable. Ses paroles en forme d'énigme sont exhibées par la jeune femme jusqu'à ce qu'elle soit elle-même assassinée : «Chaka m'a dit : "Souvent le destin improvise. Je vais faire comme lui.” Chaka, dis-moi pourquoi ? Chaka! Chaka- 
a-a-a!» (U Tam'si 1977 : 81). L'expression du deuil, le refus de manger, se résument dans cette interrogation. De personnage, Noliwé se fait écho, figure, elle réverbère le crime de son mari et le révèle de façon cryptée à son entourage. Sa folie la prive de toute intériorité et elle n'est plus, suivant l'expression de Xavier Garnier, qu'une caisse de résonance, un espace "aussi vide que le fût d'un canon» (Garnier 2001: 14). La question de Chaka et sa répétition la vident de sa substance.

L'action du roi, elle, est de plus en plus guidée par les deux syntagmes cités plus haut. Cette fois, la formule n'est plus seulement répétée par un personnage présent sur scène, elle se détache de tout locuteur identifiable pour se réduire à une voix off: «VOIX en chœur : Tue-moi, ton propre sang t'étouffera » (U Tam'si 1977 : 94). À la page suivante, seule la moitié de cette phrase est répétée selon le même procédé : «VOIX : [...] Ton propre sang t'étouffera » (Ibid.: 95). Qu'il s'agisse d'une hallucination ou d'une vision surnaturelle, l'effet est le même pour le spectateur. La mise en scène nous montre un personnage manipulé de l'extérieur qui, même s'il se prend pour le destin, est agi par des forces qui le dépassent.

Le rôle structurant des formules oraculaires - Tchicaya parlerait de "verbe »accentue la dimension poétique de cette pièce qui, par bien des aspects, se rapproche du poème dramatique de Senghor. La circulation de la parole et le travail de citation des personnages les uns par les autres amènent le spectateur-lecteur à se concentrer sur le principal matériau qui lui est fourni, à savoir un langage imagé et souvent trompeur. L'oracle ne ment pas, Chaka est bien étouffé par son propre sang, celui de ses demi-frères et non de sa mère ou d'un quelconque fils. Les signes sont là, disponibles, mais ils ne sont pas lisibles pour les principaux intéressés.

Le glissement qui s'effectue du roman à la pièce de théâtre en passant par le feuilleton nous montre des personnages qui se détachent d'une vision judéo-chrétienne du péché pour s'enferrer dans un réseau de signes et de paroles. Dans ce contexte, le concept de personnage-figure permet de considérer les acteurs présents sur scène comme des vecteurs, des supports qui donnent à voir les forces à l'œuvre dans le texte.

\section{Conclusion}

La multiplication des supports crée une constellation d'œuvres propice à l'observation et à l'interprétation. Les différents visages de Chaka donnent ainsi à voir la multiplicité des approches sur le continent mais aussi une volonté de dégager une logique globale et des héros panafricains. En proposant une vision fidèle du roman de Mofolo dans son feuilleton radiophonique, puis en s'appropriant la légende pour en faire le reflet de ses questionnements politiques, Tchicaya U Tam'si contribue à faire de Chaka une figure du dirigeant postcolonial, de ses ambitions et de ses excès. La dédicace à Senghor en tête du Zulu indique en outre qu'il prend également en considération le poème de son illustre prédécesseur.

Le feuilleton radiophonique apparaît en somme comme un laboratoire, un espace d'expérimentation pour le jeune poète. Il explore notamment l'héritage de l'orature et la question de la mémoire essentielle dans la culture traditionnelle. Le cadre de la radiodiffusion de la France d'Outre-Mer instaure un lien de communication privilégié entre Paris et les colonies, entre conteur et auditeurs, en ce sens que dans l'émission Ici Paris, courrier africain les auditeurs peuvent envoyer des contes. Pour autant, Tchicaya est bien conscient que la situation d'énonciation à la radio s'éloigne considérablement 
de celle de la veillée en cela qu'écriture, montage et enregistrement fixent une œuvre reproductible à l'infini.

On perçoit dans ces dispositifs une nostalgie qui refera surface dans l'anthologie et les recueils de nouvelles ultérieurs. Comme l'indique l'auteur dans son introduction aux Légendes : «Il ne m'est pas difficile de me souvenir des veillées d'enfance qui furent en fait ma première école » (U Tam'si 1967 : 13).

\section{BIBLIOGRAPHIE}

Afan, Huenumadji, 2006, L'Évangile Chaka, Paris, L'Harmattan.

Anta Kâ, Abdou, 1972, La Fille des dieux, Les Amazoulous, Pinthioum Fann, Gouverneur de la rosée, Paris, Présence Africaine.

Beti, Mongo et Tobner, Odile, 1989, Dictionnaire de la négritude, Paris, L'Harmattan.

Brest, René et Weitzmann, Henri, 1963, Lucien de Rubempré, tricheur romantique, feuilleton radiophonique, archive INA.

Burness, Donald, 1976, Shaka, King of the Zulus, in African literature, Washington, Three Continents Press.

Cope, Trevor, 1968, Izibongo, Zulu Praise Poems, Oxford, Clarendon Press.

Fall, Marouba, 1979, Chaka ou le Roi visionnaire, Dakar, Nouvelles éditions africaines.

Faure, William C., 1986, Shaka Zulu, South African Broadcasting Corporation, durée totale, dix heures.

Fynn, Henry Francis, 2004, Chaka roi des Zoulous, traduit de l'anglais par Estelle Henry-Bossonney, Toulouse, Anacharsis.

Garnier, Xavier, 2001, L'Éclat de la figure, étude sur l'anti-personnage de roman, Bruxelles, PIE-Peter Lang.

Gével, Claude, 1959, Vidocq, feuilleton radiophonique en 20 épisodes d'après les ouvrages de Jean Savant, archive INA.

Mabana, Kahiudi Claver, 2002, Des transpositions francophones du mythe de Chaka, Bern, Peter Lang. McMenemy, Nickie, 1973, Assegai!, Londres, Macmillan London Limited.

Mofolo, Thomas, 1931, Chaka, an Historical Romance, Translated from the Original Sesuto by F.H. Dutton, Oxford, International Institute of African languages and cultures.

Mofolo, Thomas, [1940] 2010, Chaka, une épopée bantoue, traduit directement de la langue souto par V. Ellenberger, Paris, Gallimard, coll. « Imaginaires ».

Niane, Djibril Tamsir, 1971, Sikasso ou la Dernière Citadelle, suivi de Chaka, Honfleur, P. J. Oswald. Senghor, Léopold Sédar, [1951] 2007, « Chaka », dans P. Brunel (dir.), Poésies complètes, Paris, CNRS éditions. 
U Tam'si, Tchicaya, 1967, Légendes africaines, Paris, Seghers.

U Tam'si, Tchicaya, 1977, Le Zulu, Paris, Nubia.

Vincent, Michel, 1994, Le Monde romanesque de Tchicaya U Tam'si, Ivry-sur-Seine, Éditions Nouvelles du Sud.

Zinsou, Sénouvo Agbota, [1975] 1984, On joue la comédie, Lomé, Éditions Haho.

\section{NOTES}

1. Entretien radiophonique de 1968 réalisé à l'occasion de la republication de Légendes africaines, archives INA.

2. La fiche présentant le fichier numérisé annonce un feuilleton en vingt-quatre épisodes. Cette information est confirmée à l'écoute de l'ultime segment de l'enregistrement dans lequel le présentateur précise qu'il s'agit du "vingt-quatrième et dernier épisode ». Sur cet ensemble, seulement dix-huit épisodes ont été conservés. Les deux premiers et quatre autres qu'il est difficile de situer (à partir du quatrième épisode, le présentateur ne précise plus la numérotation) sont manquants, ce qui ne nuit pas outre mesure à la compréhension de la diégèse. Le « résumé des épisodes précédents » sert en quelque sorte à combler les vides pour l'auditeur.

3. Le poème «Chaka » de Senghor est tout d'abord paru dans le numéro 12 de la revue Présence Africaine (1951) avant d'être intégré au recueil Éthiopiques en 1956 (Paris, Seuil). Dans la suite de cette étude, nous citerons l'édition des Poésies complètes publiée sous la direction de Pierre Brunel (Paris, CNRS éditions, 2007).

4. Il s'agit d'une lettre de Tchicaya à l'auteur du 18 juillet 1985.

5. Dans cet ouvrage, l'auteur oppose notamment une légende noire représentée par Thomas Mofolo et une légende dorée que l'on trouve dans le poème de Senghor. Chez ce dernier, le caractère détestable du héros est gommé pour mieux faire ressortir le portrait d'un chef trahi par les siens.

6. "But the Shaka myth in African literature is not homogenous. This is evident from the fact that the Zulu King is seen primarily as a romantic figure in Francophone Africa, as a military figure in South Africa, and is all but unknown in East Africa and in among the Onitsha Market pamphleteers of Nigeria. » (Nous traduisons.)

7. Mofolo, Thomas, Chaka, une épopée bantoue, traduit directement de la langue souto par V. Ellenberger, Paris, Gallimard, 1940. Pour toutes les citations ultérieures, nous utiliserons la réédition de 2010 dans la collection « Imaginaires » Gallimard.

8. Dans son ouvrage L'Éclat de la figure, étude sur l'anti-personnage de roman (2001), Xavier Garnier définit le personnage-figure comme un type de personnage sans véritable intériorité qui se résume parfois à une silhouette et se construit généralement par la rumeur : "Loin d'être un signe, qui escamote la force, le personnage-figure serait une force, qu'il s'agirait de penser comme telle, et dont l'impact sur le récit donnerait forme à celui-ci. » (p. 12).

9. Notons tout de même que le poème de Senghor intègre lui-aussi une "Voix blanche » qui développe un réquisitoire contre Chaka.

10. Mabana (2002: 124) : «L'intrigue est construite de telle sorte que le suicide du héros semble être le sort fatal réservé à celui qui compromet le bonheur de son peuple. »

11. Voir par exemple, Fall (1979).

12. Le feuilleton de Tchicaya a été produit et diffusé par la la Radiodiffusion d'Outre-Mer et on retrouve l'intérêt pour l'orature et les coutumes africaines dans une autre émission co-animée par Tchicaya de 1957 à 1960 - Ici Paris, courrier africain - dans laquelle les auditeurs pouvaient envoyer des contes qui étaient ensuite diffusés. 
13. La formulation dans le texte de Mofolo ([1940] 2010 : 23) est légèrement différente : «Un fils t'est né, un bœuf destiné aux vautours. »

14. Ibid.

\section{RÉSUMÉS}

Le roi Chaka (1788-1828), fondateur de l'Empire zoulou, a inspiré de nombreux auteurs africains, tant anglophones que francophones. Parmi toutes ces mises en fiction de l'histoire, le cas du Congolais Tchicaya U Tam'si (1931-1988) est particulièrement intéressant car il reprend un roman sud-africain de Thomas Mofolo et l'adapte, dans un premier temps, en feuilleton radiophonique (1957). Ce n'est que vingt ans plus tard (1976), qu'il fait jouer à Avignon sa pièce Le Zulu, qui s'éloigne considérablement de son modèle. Quoique peu connue et peu étudiée, la création radiophonique apparaît comme une étape essentielle du processus de création et de l'appropriation de cette figure par un auteur francophone majeur.

King Shaka, the founding father of the Zulu empire, has been an inspiration for African authors, whether they write in French or in English. Among all those rewritings of History, the Congolese poet Tchicaya U Tam'si stands out as he bases his work on a South African novel by Thomas Mofolo to write first a radio series (1957), then an actual play, Le Zulu, performed in Avignon twenty years later (1976). Although Tchicaya's work for the radio has not got much attention yet, it seems to be an important part of the creative process. This serialization of the novel gives us a unique insight into the appropriation of the character by a major francophone writer.

\section{INDEX}

Mots-clés : Chaka, Thomas Mofolo, Tchicaya U Tam'si, radio, feuilleton, Afrique, littérature, Southo, Congo, Afrique du Sud, missionnaires, théâtre, roman, intertextualité, réécriture, critique génétique

Keywords : Chaka, Thomas Mofolo, Tchicaya U Tam'si, radio, series, literature, South Africa, missionaries, drama, novel, rewriting, genetic criticism

\section{AUTEUR}

\section{PIERRE LEROUX}

Université Sorbonne nouvelle - Paris 3, Sorbonne Paris Cité 